\title{
Perceptions of midwifery students, mentors, and supervisors on the use of clinical teaching portfolio in Rwanda
}

Donatilla Mukamana*1, Mieke Embo ${ }^{2,3}$, Olive Tengera ${ }^{1}$, Alice Muhayimana ${ }^{1}$, Josephine Murekezi ${ }^{4}$, Hilde DeGrave ${ }^{2}$, Jean Pierre Ndayisenga ${ }^{1,5}$, Martin Valcke ${ }^{3}$

${ }^{1}$ School of Nursing and Midwifery, University of Rwanda, Kigali, Rwanda

${ }^{2}$ University College of Applied Sciences, Ghent, Belgium

${ }^{3}$ University of Ghent, Belgium

${ }^{4}$ King Faisal Hospital, Kigali, Rwanda

${ }^{5}$ Arthur Labatt Family School of Nursing, Western University, London, Canada

Received: May 15, 2020

DOI: $10.5430 /$ jnep.v10n10p52
Accepted: June 16, 2020

Online Published: July 5, 2020

URL: https://doi.org/10.5430/jnep.v10n10p52

\begin{abstract}
Background and objective: The clinical teaching portfolio is a practical tool for integrating theory and practice towards the growth of midwifery students. Both paper-based portfolios (PBP) and electronic portfolios (EP) are known and recognized as tools that can assist midwifery educators to develop students' analytical and critical thinking. Hence, midwives are required to maintain a professional portfolio to reflect their development of knowledge, skills, and attitude. The aim of this study was to explore the perceptions of midwifery students, mentors, and supervisors about the use of paper-based and electronic clinical teaching portfolios.

Methods: A qualitative descriptive design was carried out with 20 participants including midwifery students, mentors, and supervisors. Using purposive sampling, participants were recruited from two selected clinical teaching hospitals. All interviews were audio-recorded and transcribed verbatim by the research team members. Six steps of thematic analysis were followed during the inductive analysis of collected data.

Results: Three themes emerged from data analyses which include clinical teaching and learning, encountering barriers in the use of the clinical teaching portfolio, and preference among the clinical teaching portfolio users.

Conclusions: The findings of this study highlighted the importance of a clinical teaching portfolio in the promotion of midwifery clinical teaching. This study indicated that there is a need to respect the student-clinical instructor ratio and to equip the users with the required skills in order to fully benefit from the use of a clinical teaching portfolio.
\end{abstract}

Key Words: Paper-based, Electronic, Clinical teaching, Portfolio, Midwifery

\section{BACKGROUND}

Universities need to develop critically reflective, lifelong learners. ${ }^{[1]}$ In addition, registered midwives are required to keep records of evidence showing students' lifelong learning journey and to demonstrate their competences to practice. ${ }^{[2]}$ The collection of these pieces of evidence is called a portfolio

\footnotetext{
*Correspondence: Donatilla Mukamana; Email: donatillamu1@gmail.com; Address: School of Nursing and Midwifery, University of Rwanda, Kigali, Rwanda. 
which is frequently submitted in compiled printed papers. ${ }^{[3]}$ A clinical teaching portfolio is a practical methodology integrating theory and practice of the growth of midwifery students. ${ }^{[4]}$

In Sub-Saharan Africa, both paper-based portfolios (PBP) and electronic-portfolios (EP) are common, although there is limited evidence for EP. For example, in South Africa a study conducted in Western Cape University revealed that a PBP is recognized as a tool that can help midwifery educators to develop students' analytical and critical thinking. ${ }^{[5]}$ In Kenya, since the introduction of online learning, EP have become more popular than PBP with the benefit that reflection is integrated into EP. ${ }^{[6]}$ Midwives are compulsorily required to maintain a professional portfolio to reflect their development of knowledge, skills, and attitude. ${ }^{[6]}$ In addition, EP was found to be a powerful tool to develop students' communication and assist them in producing their own reflective written products and foster active learning. ${ }^{[7]}$

Training clinically competent midwives contributes positively to the reduction of maternal, newborn, and child mortality. Since 2005 in Rwanda, the maternal mortality ratio has fallen by $72 \%$ and currently stands at 210 deaths per 100,000 live births; the mortality of children under the age of five has decreased from 152 per 1,000 live births in 2005 to 50 per 1,000 live births in 2015. Despite significant progress, Rwanda continues to face a substantial burden of neonatal morbidity and mortality. ${ }^{[8]}$ Notably, neonates accounted for $39 \%$ of all deaths among children under five in $2011 .{ }^{[9]}$ As the density of midwives has been found to be positively associated with immunization coverage and maternal, infant, and child survival. ${ }^{[10]}$ It is important to strengthen the education of future midwives so that more competent midwives can help to achieve the Rwandese healthcare objectives.

The shortage of health professionals in Rwanda, including limited numbers of midwife experts in clinical teaching resulted in a theory-practice gap. Although many strategies are being used to boost clinical teaching in midwifery, the theory-practice gap and inconsistency of clinical expectations between classroom and clinical practice settings is still required to be addressed. ${ }^{[9]}$

Very little is known about the use of the portfolio in clinical teaching and learning in Rwanda. Therefore, this article aims to explore the perceptions of midwifery students, mentors, and supervisors on the use of the clinical teaching portfolio with the following specific objectives:

1) Describe the perceptions of midwifery students, mentors, and supervisors on the use of the clinical teaching paperbased portfolio;
2) Describe the perceptions of midwifery students, mentors, and supervisors on the use of the clinical teaching electronic portfolio; and

3) Compare both paper-based and electronic clinical teaching portfolios.

\section{METHODS}

\subsection{Study design}

A descriptive qualitative design was used to explore the perceptions of midwifery students, mentors, and supervisors on the use of the clinical teaching portfolio. The study was conducted in Obstetrics and Gynecology (OBGY) departments for two selected clinical teaching hospitals in Rwanda.

\subsection{Study population}

The study was conducted with midwifery undergraduate students, supervisors and mentors who have been in clinical placement at two selected clinical teaching hospitals and who have used both PBP and EP in their clinical teaching and learning activities regularly since the beginning of 2019 to the time of data collection.

\subsection{Sampling}

The purposive sampling strategy ${ }^{[11]}$ was used to select participants who could provide rich information about their perceptions of the use of a portfolio in their clinical teaching and learning practices in Rwanda. Between the period of May to October 2019, a total of 20 participants including twelve midwifery students, four supervisors from midwifery departments, and four mentors from two selected clinical teaching hospitals enrolled in the Medbook platform were involved in this study. The Medbook platform was used by all participants depending on their roles. Students entered their daily reflections based on their clinical learning goals which are in line with existing International Confederation of Midwives (ICM) competences. Therefore, mentors and supervisors provided the feedback to the students using the same platform.

Participants were contacted by the research team members after providing them with the letter of information introducing the study and inviting them to participate in this study. Inclusion criteria included being a midwifery student, supervisor, and/or mentor allocated at two selected clinical teaching hospitals, exposed to the existing PBP and initiated EP with a willingness to be part of the study. Midwifery students, mentors, and supervisors who were not enrolled in Medbook and who were not exposed to existing PBP or EP were excluded. 


\subsection{Data collection}

The data collection was carried out in two steps. The first step included data that were collected at the beginning of clinical placement where participants shared their perception on the use of PBP before being exposed to EP. For the second step, data were collected at the end of the clinical placement where participants shared their perception on the use of EP. In addition, to capture the similarities and differences between PBP and EP, participants were asked to compare them from their own experiences and perspectives.

Using a semi-structured interview guide and demographic questionnaire, in-depth individual interviews of 45 to 60 minutes were carried out with midwifery students, mentors, and supervisors. Participants signed consent immediately prior to starting the interview. Given that all participants were able to communicate fluently in English as an academic language, all correspondence and interviews were in English. In this study the interviews were recorded after obtaining permission from participants. To elicit participants to express themselves freely, a sample of the used probing questions were used. All recorded interviews were transcribed verbatim by the research team members. Demographic information of participants was collected using a demographic form prior to the start of in-depth individual interviews.

\subsection{Trustworthiness}

To achieve and maintain the rigor and quality of this study, the research team carefully considered four dimensions of trustworthiness including credibility, confirmability, dependability, and transferability. ${ }^{[12]}$ Credibility was ensured by correctly identifying eligible participants for the study. In order to gain credible data, the interviewers were guided by a pre-developed, semi-structured interview guide composed of open-ended questions to allow participants to share their perceptions on the use of a clinical teaching portfolio with clear and detailed descriptions. In addition, the principal investigator trained the research team and each team member engaged in pilot interviews to ensure that they were able to collect credible, consistent data. Furthermore, peer debriefing among research team members was used during the data collection and analysis process to maintain the credibility of collected data.

To ensure dependability, research team members recorded all decisions made from the beginning of the research process and during data collection to ensure that all interviews were conducted under similar situations. In addition, the principal investigator ensured that there was regular collaboration among research team members so that recruitment followed pre-established inclusion criteria. Guided by the principal investigator, all steps of data analysis were monitored regu- larly on a weekly basis. For confirmability, the research team members ensured that reflective and memo notes were taken during the data collection and analysis process to ensure that all feelings, impressions, and thoughts were captured during the time of data analysis. Also, the research team agreed on the final themes and sub-themes which emerged from data analysis to ensure that they were representative of participants' perceptions. To achieve transferability, detailed descriptions of the research processes and participants including sampling strategies, inclusion criteria, the data collection process, and participants' demographic information were provided. Also, direct quotes from participants' perceptions were used. In addition to the four dimensions of trustworthiness, reflexivity was used by research team members to acknowledge their own role, assumptions, and bias in this research process, especially during data collection, analysis, and interpretation. ${ }^{[13]}$ Also, in this study, reflexive notes for research team members included the descriptions of study settings, aspect and conditions of conducted interviews and researchers' reflections arising during the transcription and analysis of transcribed data. Researchers' reflexive notes also included the description of their relationship with participants.

\subsection{Data analysis}

Thematic Analysis guided the process of analyzing data step by step and the perceptions of using the clinical portfolio was understood and well described. ${ }^{[14]}$ Data were analyzed in two phases. The first phase was related to the information collected on the use of PBP. The second phase began when data on the use of EP in clinical settings were gathered. The process of data analysis lasted for a period of 4 months starting from June 19, 2019 to November 07, 2019. The first session conducted in June was dedicated to the learning process, analyzing qualitative data focused mainly on thematic analysis. This approach was guided by Braun and Clarke ${ }^{[15]}$ framework of thematic analysis which assisted the research team in identifying significant themes that were meaningful in addressing the research questions on the perceptions of the use of a clinical portfolio.

Six steps of thematic analysis guided by Braun and Clarke ${ }^{[16]}$ guided the data analysis. During the first step, all recorded interviews were transcribed by each member of the research team who reviewed the text more than once highlighting data that are more meaningful in addressing the research questions on the perception related to the use of a clinical teaching portfolio. The second step guided the process of generating initial codes. At this stage the research team started the coding process in a systematic way, highlighting those codes that were meaningful to the issues under study and that were 
recurrent. The third step consisted of searching for themes. While on step two the team worked separately in sorting out the codes, at stage three the team came together and agreed on cluster codes. The cluster codes were then filtered, and similar cluster codes merged to form sub-themes. At the fourth step, which was aimed at reviewing the themes, subthemes with similar meaning were grouped together and the decision was taken on which sub-themes to be kept or to be deleted. On the fifth step, themes were defined in accordance with the perceptions of midwifery students, supervisors and mentors on the use of the clinical teaching portfolio, the benefits and challenges encountered while using that tool, as well as suggestions on its further implementation in midwifery education in Rwanda. Finally, step six consisted of writing up the report.

This analysis process was supported by Microsoft Word and Microsoft Excel. As recommended by Osborg, ${ }^{[17]}$ the research team used these two Microsoft Office programs to organize the data. The transcripts that were on Word files were transferred to the Excel sheet for the coding process. This method wasn't intended to quantify qualitative data, rather to provide a more structured manner to handle the large amount of data generated from the perceptions on the use of clinical teaching portfolio by midwifery students, supervisors and mentors. Throughout the entire process the data was shared among research team members. Each member utilized the same review process individually, and then as a group determined consensus on the codes, sub-themes and themes presented in this report. This was carried out through email exchange and regular Skype calls.

\section{FINDINGS}

\subsection{Description of participants}

The study participants consisted of six male and six female midwifery students, in both Diploma and Bachelor of Midwifery programs. There were four mentors and four supervisors working in the two selected clinical teaching hospitals.

\subsection{Summary of analysis}

The three main themes which emerged from the study included improving clinical teaching and learning, identifying barriers encountered in the use of a clinical teaching portfolio, and determining the preference among clinical teaching portfolio users.

\subsubsection{Theme one: Improved clinical teaching and learn-} ing

Improved clinical teaching and learning was characterized as positive changes experienced by participants in the clinical teaching and learning process as a result of using a clinical teaching portfolio. This theme is related to the ultimate goal

Published by Sciedu Press of using a clinical teaching portfolio which is to improve the quality of midwifery pre-service education. Participants' perceptions on how the use of a clinical teaching portfolio improved their clinical teaching and learning process is described under the following three sub-themes: promote student-centered teaching and learning, enhanced students' self-directed learning, and improved clinical evaluation and monitoring of students' performance.

\section{Sub-theme: Promoted student-centered teaching and learning}

Most participants including students, mentors, and supervisors described that consistent and effective use of a clinical teaching portfolio in their clinical teaching and learning process promoted student-centered teaching and learning significantly. One student mentioned:

“..., the use of portfolio helped me to be more responsible of my clinical learning process than before as it helped me to set my clinical learning objectives and I made sure that were also achieved and presented in my portfolio and got feedback from my mentors" (Student.JN).

The promotion of student-centered clinical teaching and learning was highlighted by both mentors and supervisors. They described that midwifery students are better able to develop their independence and autonomy in their clinical learning activities, such as setting daily learning objectives and evaluating themselves and their learning outcomes regularly. One participant stated:
"When our students are using a clinical portfolio regularly, they become responsible for choosing and deciding what they will learn based on their clinical objectives and they present to me how they are planning to achieve that and together we have an agreement of how that could be assessed by me or by themselves..." (Mentor J.U).

Participants commented that students and their mentors or supervisors were able to use the clinical teaching portfolio to focus on clinical objectives and skills that enable students to become independent and lifelong learners in their clinical practices. One participant said:

"as a results of using portfolio, I am happy that clinical teaching become more student-centered than before and students are able to define the meaning of their clinical learning based on their previous experiences, new knowledge, and skills gained from clinical learning activities, literatures and from their peers, mentors and supervisors" (Supervisor.A.U). 


\section{Sub-theme: Enhanced self-directed learning}

Participants described how the use of portfolio in their clinical teaching and learning process enhanced students' selfdirected learning in the following terms:

"After giving the feedback, the learning becomes easy as it helps the students to identify their weakness and correct it with or without my presence then the learning process goes well (Supervisor E.M).

In regard to self-directed learning as a result of using a clinical teaching portfolio, one mentor indicated that: “... this helps the student to be oriented without waiting for the supervisor to come to school or the mentor who is not at school" (Mentor J.U). Most participants mentioned the clinical teaching portfolio improved students' competences in formulating their learning objectives at the beginning of their clinical learning period. As one participant stated, "Students can formulate the objectives and they reflect and then as mentors we share the feedback online" (Mentor R U).

Sub-theme: Improved clinical evaluation and monitoring of students' performance

All participants expressed that there was a concrete improvement in students' evaluation and monitoring of their clinical performance resulting in the consistent use of a teaching portfolio. Participants appreciated how a clinical teaching portfolio helped them to provide and receive feedback on time. One student stated, "Benefits are many, ehhhh like time management because time here is expressed in two ways: the time to get the feedback and the time to get the report on what I do every day" (Student A.M). Also, different participants described that the use of a clinical teaching portfolio facilitated them in their evaluation of students and in providing and receiving feedback during clinical practices on a timely basis. One supervisor mentioned:

“... easy to give feedback to different people, so for me also it is an opportunity to learn and to follow students how they are progressively achieving their clinical learning objectives rather than to wait at the end of clinical ......" (Supervisor E.M).

Participants mentioned that the use of a clinical teaching portfolio improved the manner used to monitor students' progress and performance. As stated by one mentor:

"The best benefits are that sharing capability where the supervisor and the mentor can evaluate and monitor the student's activities, achievements, and progress on the field without taking much time...., by reading the student's reflection and summarize (Mentor.M.M).

This was echoed by a participant student who stated, "When I am given feedback immediately it means I take decisions accordingly and quickly which I improve my learning speed. . . meaning I will do correct things in a short time" (Student A.M). Another benefit of a clinical teaching portfolio is ensuring the credibility of information shared by the students in their learning process. "Another benefit it's the way hospitals are involved in clinical or students' mentorship to make sure that everything the student does is credible..." (Mentor J.U)

\subsubsection{Theme two: Encountered barriers in the use of a clinical teaching portfolio}

Whether students, mentors, or supervisors, participants reported a number of barriers and challenges that limited or negatively affected their abilities to use both PBP and EP in their clinical teaching and learning process. Three subthemes emerged as barriers that participants expressed in the use of a clinical teaching portfolio including insufficient knowledge on clinical teaching portfolio users, limited resources, and heavy workload among mentors and supervisors.

\section{Sub-theme: Insufficient knowledge of clinical teaching portfolio users}

Most participants of this study declared that the effective use of the portfolio was hampered by limited knowledge and understanding about clinical teaching portfolios among users. Reflecting on this, one participant said:

"The supervisors who didn't have enough knowledge to use the clinical portfolio.... Even though some of us also don't have knowledge about it, that is the same challenge for supervisors or mentors and many students" (Student B.D).

Participants also discussed how limited knowledge becomes a significant barrier for using a clinical teaching portfolio for both mentors and supervisors while teaching students in different clinical teaching activities. One participant put it in this way:

"We met the challenge of the supervisor or mentors who are not familiar with portfolio and they are not using it in the same way and that confuses us .... they are not consistent in their guidelines of using portfolio" (Student A.M).

\section{Sub-theme: Limited resources}

Internet connection, computers and electronic devices were perceived as a big challenge which hindered the participants 
effective use of both PBP and EP. Some participants mentioned that numerous students and their clinical educators, being mentors or supervisors, do not have equal access to those basic materials and equipment. One clinical mentor stated, “...It's easy to connect myself to the EP but the machines are not enough for us and everywhere you are" (Mentor R.U). Many participants described how their willingness and readiness to use a clinical teaching portfolio were often limited by lack of or a poor internet connection which is necessary to access online materials essential for portfolio content. One supervisor stated, "Challenge is the internet connectivity and it was not easy at the site to open the network" (Supervisor E.M). Also, the issues of internet connectivity was experienced by students who were not able to access or send their completed clinical portfolio-related activities. One participant stated:

"The challenges are about the networks because when you are using clinical teaching portfolio you need the connection, and other electronic materials while preparing either PBP or EP contents or materials ... for example when you are writing reflection you need to support them with online articles" (Student E.N).

\section{Sub-theme: Heavy workload among mentors and super- visors}

Participant-mentors and supervisors commented that some working conditions were not favorable for them to balance clinical teaching and their daily assigned activities. One participant mentioned:

"as a clinical mentor... I always have a heavy workload whereby I have many other responsibilities of caring a big number of mothers in labor ward or sometimes coordinating clinical midwifery activities within my department apart from teaching students" (Mentor. R.U)

Student participants described that their supervisors and clinical mentors had many documents to be completed and that limited their time to read, revise, and focus more on the submitted student's clinical portfolio. One participant commented:

"I may think about it, it may be the time for the supervisors and mentors they have, they have many documents in their office, they have many students...100 documents in front of the supervisors" (Student. E.N).

Participants highlighted that the issue of heavy workloads Published by Sciedu Press among clinical mentors and supervisors is linked to the high student-instructor ratio.

"......sometimes when we give them feedback to students about their clinical performance and progress it is not easy because they are so many and you can imagine having a big number of them some time you provide only feedback to $20 \%$ of them means that $80 \%$ of them will get their feedback on clinical portfolio too late to late ....- it is a big problem" (Supervisor B.H).

Participants described that mentors' and supervisors' heavy workload negatively affected the process of receiving feedback, and consequently hampered the process of receiving clinical evaluation and performance feedback on a regular basis. As one participant remarked:

"it may be caused by the heavy workload of the supervisors, sometimes they delay giving us feedback, they have many documents stay in their office...., and getting feedback from them takes a long time" (Student D.N).

\subsubsection{Theme Three: Preference among Clinical Teaching Portfolio Users}

This theme was characterized by participants' perceptions about their preferences between both PBP and EP. It appears from the below statements that EP, which was described as user friendly, was preferred to PBP which was perceived as expensive and time consuming.

\section{Sub-theme: Financial cost and time consuming}

Participants shared their perception about the financial implications of using PBP which could have a significant impact on the users' preference between the two types of portfolios. One participant stated:

“.... you need materials, we need paper to write that information, those papers sometimes are at high costs. we need to print papers, no money we have, the money will go away, it is reduced in our pockets, many of us do not have the money" (Student E.N).

Participants mentioned the amount of time consumed as another factor that could influence their preference in choosing either PBP or EP. Participants expressed that compiling together all components of PBP consumes time that could be allocated to some other clinical learning activities. One mentor stated, ".... Another thing is to have time to sit and read 5-10 copies, ... that requires enough or extract time to write constructive feedback to students" (Mentor J. U). On the 
other hand, participants highlighted that even if a portfolio promotes student-centered clinical teaching, "it takes longer time" and they called PBP a tedious clinical tool. One student mentioned, "......Did you see that time to go out to print the papers and to bring it to the supervisor" (Student. D.N ).

\section{Sub-theme: User friendly}

Most participants who were involved in this study felt that EP was more user friendly when compared to PBP. In this regard, many participants reported they preferred EP as it was easy to read and to navigate compared to PBP. One participant stated, "We learnt how to make a portfolio in an easy way, in a suitable situation without struggle, without any problems and for the supervisor they give us the feedback early" (Student E.N). Supervisor E.M shared the same perceptions, "This was easy because everything is done online".

Some participants mentioned that EP demands a high level of computer-related skills and competencies, which fortunately they acquired from their ICT course. One participant said,

"I learned to introduce ICT in our usual subject because even this portfolio goes with practicing ICT because we use computers and the internet, so I experienced skills and knowledge about computers and the internet...." (Student A.M).

\section{Discussion}

The study findings revealed that both PB and EP clinical portfolios provided the benefit of improving clinical teaching and learning. It enhances student-centered learning, selfdirected learning and monitoring as well as evaluation of students. Apart from this, participants claimed a number of challenges or barriers that limited or negatively affected their abilities to effectively use both PBP and EP in their clinical teaching and learning process. Limited knowledge and understanding about clinical teaching portfolios, limited resources, and heavy work workloads were reported. Most participants appraised that EP was more user friendly than PBP. Participants argue that both clinical teaching portfolios involve financial cost. The amount of time consumed was mentioned by participants as another factor that could influence their preferences and was attributed mostly to PBP.

The results revealed that the clinical teaching portfolio promotes student-centered teaching and learning. Participants commented that they were able to use the clinical teaching portfolio to focus on clinical objectives and skills that enable students to become independent and lifelong learners in their clinical practices. Therefore, students benefit from independent clinical learning opportunities by reflecting on their previous experiences. They also reported they gained new knowledge and constructive skills from clinical learn- ing activities, including through readings, from their peers, mentors and supervisors. Similar literature stated that the ultimate role of a clinical portfolio is to integrate theory and practice in the growth of midwifery students ${ }^{[4,5,18]}$

Participants expressed that the clinical teaching portfolio makes students more responsible for their learning process. Students were able to identify their clinical learning needs and goals and collaborate with their mentors and supervisors to establish appropriate learning and evaluation strategies that will enable them to achieve their learning outcomes. Students were eager to share their perceptions on how using a clinical teaching portfolio enhanced their self-directed learning as confirmed by their supervisors and mentors. The majority of participants mentioned the clinical teaching portfolio improved students' competences in formulating their learning objectives at the beginning of their clinical learning period. Similar findings reported that use of a clinical teaching portfolio is a powerful tool to produce students' own reflective written products and foster active learning. ${ }^{[7,19]}$ Therefore, this tool facilitates the development of self-directed learning skills by the formulation of learning objectives, reflection on performed activities, self-evaluation of performance, and plan for future learning activities. ${ }^{[18,20]}$

In this study, all participants expressed a concrete improvement in students' evaluation and monitoring of their clinical performance resulting in consistent use of a clinical teaching portfolio. Students who participated in this study argue that the use of a clinical teaching portfolio was beneficial in different ways. The feedback was provided quickly, hence their time was saved and it also facilitated interactions with their facilitators. Clinical students are progressing and monitored on a daily basis. Use of a clinical teaching portfolio supports the certainty of information shared by the students in their learning activities. Indeed, midwives are required to keep records of evidence showing a student's lifelong learning journey and to demonstrate their competences to practice; this requires integration, continuity and collaboration. ${ }^{[2,21]}$

Most participants of this study claimed that the effective use of portfolios was hindered by limited knowledge and understanding about clinical teaching portfolios. Participants reported insufficient knowledge of clinical teaching portfolio users. They also discussed how limited knowledge becomes a significant barrier for using a clinical teaching portfolio for both mentors and supervisors while teaching students in different clinical teaching activities. In a similar study conducted in the United Kingdom (UK), students revealed that supervisors were inconsistent in evaluating students using portfolios. ${ }^{[22]}$ We believe that most of the time this inconsistency in assessing students is due to lack of harmonized 
training among the assessors. Limited technology skills are significant constraints for the implementation of clinical teaching portfolios.

All participants identified the shortage of resources such as internet connection, computers and electronic devices as a big challenge which hindered their effective use of clinical teaching. Some participants explained that the use of both PBP and EP required users to have essential materials such as computers, modems and external devices to store and save different electronic materials and contents of their clinical portfolio, however many students and their mentors or supervisors do not have equal access to those basic materials and equipment. Many participants including both students and their clinical educators described how their willingness and readiness to use clinical teaching portfolios were often limited or disrupted by lack of or poor internet connection which is necessary to access online materials essential for portfolio content. The issues of internet connectivity were experienced by students who were not able to access or send their completed clinical portfolio-related activities. Similar to previous studies in Sub Saharan Africa, lack of stable internet connectivity and locally developed content, unaffordable internet cost, and limited technology skills are common barriers. ${ }^{[23]}$

Participants, mentors, and supervisors commented that some working conditions were not favorable for them to balance clinical teaching and their daily assigned activities. The main factor associated with that was a heavy workload; supervisors and clinical mentors had many documents to be completed, limiting their time to read, revise, and focus more on students' submitted clinical portfolios. Participants described that mentors' and supervisors' heavy workloads negatively affected the process of providing and receiving feedback, and consequently hampered the process of clinical evaluation and monitoring students' progress and performance on a regular basis. The study conducted in Rwanda revealed that challenges in the clinical setting included the shortage of clinical supervisors, or turnover and often the absence of clinical supervision in a severely resource-constrained environment. ${ }^{[9]}$

In this study, most participants reported that EP was more user friendly (easy to read and to navigate, easily accessible everywhere) when compared to PBP. This is similar to the findings from Pincombe ${ }^{[24]}$ where students said that EP is simple, organized, and safe while PBP is not easy and not clear. Similarly, another study conducted in South Africa revealed that $\mathrm{EP}$ is more user-friendly and less cumbersome. ${ }^{[25]}$

Participants argue that PBP involves more financial cost than EP due to the large amount of paper to be printed. Most participants considered EP less expensive than PBP. Hence, participants mentioned that the cost of a clinical teaching portfolio can be higher depending on printing portfolio materials and binding them before being submitted to the clinical mentors. In the study done by Brennan and Lennie, ${ }^{[22]}$ the majority of students reported that they are required to print out a huge amount of paper.

Participants expressed that compiling together all components of a clinical teaching portfolio consumes significant time that could be allocated to other clinical learning activities, making it harder for them to effectively achieve their clinical learning objectives. PBP has been perceived by the students as a tiresome tool and more time consuming than EP. The study done by Buzzetto-More ${ }^{[26]}$ reported that students spent more time on PBP. As in the study done by [25] students spend more time binding the papers and experience anxiety about losing those papers.

\subsection{Strengths and limitations}

To our knowledge, this is the first study to investigate the perceptions of midwifery students, mentors, and supervisors of the use of clinical teaching portfolios (PBP and $\mathrm{EP}$ ) in Rwanda. Using qualitative description design helped the researchers to provide a rich description of these perceptions and experiences of using clinical teaching portfolios in Rwanda. Therefore, the findings from this study contribute to the limited body of literature in midwifery education in Rwanda as well as in the region. However, this study has some limitations related to settings and populations that deserve to be mentioned. Although other health professionals including nurses and physicians are using portfolios in their clinical teaching and learning activities, only midwifery students and supervisors from one teaching institution and mentors from only two clinical teaching hospitals participated in this study. This highlights the need to involve other health professionals from other academic and clinical settings that are using portfolios in their teaching and learning activities in future studies.

\subsection{Implication and recommendations}

To strengthen the quality of midwifery education, a clinical teaching portfolio could be used to promote student-centered clinical teaching. The study findings showed that there is a need to maintain the consistency for the use of clinical teaching portfolio tools and for training clinical teaching portfolio users. The midwifery department in collaboration with stakeholders may provide necessary materials required for effective use of clinical teaching portfolios at the beginning and during the clinical period.

For further studies, other methods such as statistical results 
from the quantitative approaches may complete the qualitative findings and help to understand how different factors affected the use of a clinical teaching portfolio.

\section{Conclusion}

Clinical teaching portfolios are viewed as an important tool that improves the clinical teaching and learning processes for midwifery students. The continuous exchange between students and clinical educators is the cornerstone in building the competencies for a capable midwifery student. The success of a clinical teaching portfolio depends on the availability of knowledgeable, mentors and supervisors, and sufficient equipment for the teaching and learning process.

\section{ACKNOWLEDGEMENTS}

The authors acknowledge VLIR/UOS for financial support and Imengine company (Medbook) for the e-portfolio platform.

\section{CONFlicts OF INTEREST Disclosure}

Authors declare that it is not allowed to transform, copy or use this document illegally in any way without the author's authorization.

\section{REFERENCES}

[1] Kwok LF, Hui YK. The role of e-portfolio for smart life long learning. Smart Innov Syst Technol. 2018. https : //doi.org/10.1007/97 8-3-319-59454-5_11

[2] Birks M, Hartin P, Woods C, et al. Students' perceptions of the use of eportfolios in nursing and midwifery education. Nurse Educ Pract. PMid:27235565 https ://doi.org/10.1016/j.nepr.2016.03 .003

[3] Haggerty C, Thompson T (2017) The challenges of incorporating ePortfolio into an undergraduate nursing programme. Open Prax. https://doi.org/10.5944/openpraxis.9.2.554

[4] Mollahadi M, Khademolhoseini SM, Mokhtari-Nouri J, et al. The portfolio as a tool for mentoring in nursing students: A scoping review. Iran J Nurs Midwifery Res. 2018. PMid:30034481 https : //doi.org/10.4103/ijnmr. IJNMR_195_17

[5] Ticha V, Fakude LP. Reflections on clinical practice whilst developing a portfolio of evidence: Perceptions of undergraduate nursing students in the Western Cape, South Africa. Curationis 38: 1-8.

[6] Priscah MJ, Ronald OO, Tecla SJ. Portfolio development as a method of learning, assessment and evaluation in clinical Nursing Education in Kenya. Int. J. Sci. Res. Innov. Technol. 2016.

[7] Gaba A. Development and Evaluation of an e-portfolio for Use in a Dietetic Internship Program. Procedia - Soc Behav Sci. 2015 https://doi.org/10.1016/j.sbspro.2015.01.731

[8] National Institute of Statistics of Rwanda, Rwanda Ministry of Health and II 2015 (2013) Rwanda Demographic and Health Survey 2014 15: Key Indicators. Rockville, Maryland, USA: NISR, MOH, and ICF International. J Chem Inf Model. https : //doi .org/10.101 7/CB09781107415324.004

[9] Uwizeye G, Mukamana D, Relf M, et al. Building Nursing and Midwifery Capacity Through Rwanda's Human Resources for Health Program. J Transcult Nurs. 2017.

[10] Anand S, Bärnighausen T. Health workers and vaccination coverage in developing countries: an econometric analysis. Lancet. 2007 https ://doi.org/10.1016/S0140-6736(07)60599-6

[11] Sandelowski M. Focus on qualitative methods: Time and qualitative research. Res Nurs Heal. 1999. https://doi.org/10.1002/(SI CI) 1098-240X (199902) $22: 1<79:$ : AID-NUR9>3 . 0 . CO;2-3

[12] Lincoln YS, Guba EG. Establishing Trustworthiness. Nat. Inq. 1985.

[13] Mauthner NS, Doucet A. Reflexive accounts and accounts of reflexivity in qualitative data analysis. Sociology. 2003. https ://doi.or g/10.1177/00380385030373002
[14] Maguire M, Delahunt B. Doing a Thematic Analysis: A Practical, Step-by-Step Guide for Learning and Teaching Scholars. All Ireland Journal of Teaching and Learning in Higher Education. All Irel. J. Teach. Learn. High. Educ. 2017.

[15] Braun V, Clarke V. Teaching thematic analysis: Overcoming challenges and developing strategies for effective learning. Psychologist. 2013.

[16] Braun V, Clarke V. Using thematic analysis in psychology. Qual Res Psychol. 2016; 3: 77-101. https://doi.org/10.1191/147808 8706qp063oa

[17] Ose SO. Using Excel and Word to Structure Qualitative Data. J Appl Soc Sci. 2016. https : //doi .org/10.1177/1936724416664948

[18] Green J, Wyllie A, Jackson D. Electronic portfolios in nursing education: A review of the literature. Nurse Educ Pract. 2014. PMid:24090523 https://doi.org/10.1016/j.nepr.2013.08 .011

[19] Nielsen K, Pedersen BD, Helms NH. EPortfolio and learning styles in clinical nursing education. J Nurs Educ Pract. 2015. https: //doi.org/10.5430/jnep.v5n9p54

[20] Beckers J. With a little help from my peers. Int Conf Intell User Interfaces, Proc IUI. 2016. https : //doi .org/10.1145/337732 5.3377518

[21] Embo M, Valcke M. Workplace learning in midwifery education in Flanders (Belgium). Midwifery. 2016. PMid:26747208 https : //doi.org/10.1016/j.midw.2015.11.021

[22] Brennan KM, Lennie SC. Students' experiences and perceptions of the use of portfolios in UK preregistration dietetic placements: A questionnaire-based study. J Hum Nutr Diet. 2010. PMid:20113384 https://doi.org/10.1111/j.1365-277X.2009.01028.x

[23] Luera G, Brunvand S, Marra T. Challenges and Rewards of Implementing ePortfolios through a Bottom-Up Approach. Int. J. ePortfolio. 2016.

[24] Pincombe J, McKellar L, Weise M, et al. ePortfolio in Midwifery practice: "The way of the future." Women and Birth. 2010. PMid:19523891 https://doi.org/10.1016/j.wombi.2009.0 5.001

[25] De Swardt M, Jenkins LS, Von Pressentin KB, et al. Implementing and evaluating an e-portfolio for postgraduate family medicine training in the Western Cape, South Africa. BMC Med Educ. 2019. PMid:31286945 https : //doi.org/10.1186/s12909-019-169 $2-x$

[26] A. Buzzetto-More N. Assessing the Efficacy and Effectiveness of an E-Portfolio Used for Summative Assessment. Interdiscip J e-Skills Lifelong Learn. 2010. https : //doi .org/10. 28945/1164 